\title{
PRESUPUESTOS ONTOLÓGICOS PARA LA DECLARACIÓN UNIVERSAL DE LOS DERECHOS DE LA MADRE TIERRA ${ }^{1}$
}

\author{
OMAR FELIPE GIRALDO
}

\begin{abstract}
Recibido el 10 de abril de 2012 y aprobado el 20 de agosto de 2012
\end{abstract}

\section{RESUMEN}

El Buen Vivir es una utopía que surge en Latinoamérica inspirada en las racionalidades de los pueblos indígenas, campesinos y afro del subcontinente la cual, a contracorriente de los discursos hegemónicos, está teniendo una enorme influencia en las discusiones mundiales sobre la gran crisis civilizatoria. Específicamente, en el presente artículo analizamos el proyecto de declaración universal de los derechos de la Madre Tierra, considerando las bases ontológicas que le dan sustento. El objetivo es comprender la pregunta que interroga por el sentido del ser a través de respuestas en torno a la relacionalidad con el todo, así como discutir la relevancia de considerar estas ontologías en un momento de la historia en donde nos estamos cuestionando por la continuidad de la vida humana en el planeta.

\section{PALABRAS}

CLAVE

Derechos de la naturaleza, buen vivir, ontología relacional, crisis civilizatoria

ONTOLOGICAL ASSUMPTIONS FOR THE UNIVERSAL
DECLARATION OF THE MOTHER EARTH'S RIGHTS

\section{ABSTRACT}

The "Buen Vivir" is a utopia that arises in Latin America inspired by the rationalities of indigenous peoples, peasants and afro-descendant people of the subcontinent which, against the current hegemonic discourses, is having a huge influence in global discussions about the civilization crisis. The purpose of this paper is to analyze the universal declaration of Mother Earth's rights draft, considering the ontological foundations that support it. The aim is to understand the question asks about the sense of being through answers around the relationality to the whole, as well as to discuss the relevance of considering these ontological assumptions in a moment of history in which we are challenged by the continuity of human life on the planet. 


\section{KEY WORDS}

Rights of nature, buen vivir, relational ontology, civilization crisis

\section{INTRODUCCIÓN}

Uno de los aspectos determinantes y reproductores de la actual crisis civilizatoria es la miseria del pensamiento. No nos estamos refiriendo a la estrechez de un pensamiento que pueda expresarse en nuevas realizaciones científicas y técnicas, sino que hablamos de la pobreza de un pensar que reconozca, desde una perspectiva ontológica, la amenaza que para la vida ha ocasionado la obra predatoria de la sociedad moderna. Efectivamente, la humanidad enceguecida por su capacidad de controlar, manipular y dominar la naturaleza a su antojo, huye ante la pregunta que hoy es más importante: aquella que interroga por la posibilidad de seguir siendo en el mundo, es decir, la cuestión de que su especie no desaparezca del planeta. El problema es que este cuestionamiento no puede solucionarse a través de un pensamiento técnico del hacer y el fabricar, pues se trata de un problema eminentemente ontológico que debe resolverse a través de respuestas que atiendan al ser en cuanto ser, y no por referencia a lo cósico, como fue ampliamente analizado por Heidegger en su obra El Ser y el Tiempo (1971).

La pregunta acerca del dilema de la supervivencia de la especie humana en el planeta, es el objeto del discurso que quizá con mayor fuerza ha adquirido el estatuto para ser discutido en el comienzo del siglo XXI. En torno a dicho tema se ha construido un discurso dominante, el cual sostiene que la debacle ambiental a la que hoy nos enfrentamos puede ser remediada con el desarrollo e implantación de tecnologías más amigables con el medio, y con un capitalismo verde responsable y respetuoso con el entorno. Sin embargo, la pregunta a la que estamos emplazados a contestar interroga por el sentido del ser en el mundo, por lo que la solución a la pregunta tiene que darse en la dirección ontológica en la que la misma pregunta está hecha. No es nuestra intención decir que debamos quedarnos en una abstracción filosófica. Más bien queremos expresar que si lo que está en peligro es la continuidad de la vida humana $-\mathrm{y}$ no decimos la totalidad de la vida en la Tierra-, lo que debe repensarse es la vida desde una perspectiva existenciaria ${ }^{3}$ y desde ahí plantear soluciones pragmáticas más creativas para enfrentar la crisis civilizatoria contemporánea. 
Así entonces, la miseria del pensamiento a la que aludimos es el resultado de la concepción occidental del ser humano como un ente individualizado, separado de la naturaleza, en la que él se concibe a sí mismo en el centro sobre el cual se fundamenta todo lo existente (Heidegger, 1996). Bajo ésta premisa ontológica construida durante la modernidad, la humanidad se ha dirigido hacia el mundo con la ambición de poseer y dominar (Adorno y Horkeimmer, 1987). Resulta lógico que ahora, durante la devastación ambiental, pretenda seguir sojuzgando al mundo, y continúe creyendo que la salvación de su especie pueda darse a través de un cambio en el hacer técnico, sin considerar antes la pregunta por su ser. No es que consideremos que la técnica no sea un instrumento valioso. Lo es y no podemos prescindir de ella. La cuestión es que al concentrarnos en la sujeción del medio, hemos olvidado el sentido de nuestro ser, y, entretanto, corremos el peligro de ya no continuar siendo posibles.

La percepción del ser humano como el único sujeto sobre la Tierra, y todo lo demás como vulgares objetos disponibles para nuestros afanes explotadores, es la racionalidad antropocéntrica que hemos heredado de la modernidad colonial la mayor parte de los pueblos del mundo. No se trata de la totalidad de concepciones ontológicas, sino la de una muy particular forma de autopercepción fragmentada, que desliga, compartimenta, y desune lo que inmanentemente está unido. Corresponde a una forma muy específica de aprehender al ser, derivada de un saber que ofrece la estructura de significaciones para que el capitalismo pueda sustentarse. Ciertamente, para acometer el soberbio proyecto de convertirnos en amos y señores de la naturaleza, es imprescindible imaginarnos antes en seres autónomos, independientes y autárquicos del entorno, pues sería imposible dominar la Tierra si tenemos conciencia de ser una de sus partes constitutivas. De modo que la miseria del pensamiento consiste en el olvido de que la condición de nuestra existencia no es la dominación, sino la relación con otros sujetos naturales, puesto que la posibilidad de su existencia es el requisito ineluctable para la posibilidad de nuestra vida.

La construcción de tales racionalidades no constituyen un efecto perverso de la modernidad que sería necesario corregir como lo argumentarían sus defensores; antes bien, es la esencia misma del proyecto, que necesitó del avasallamiento de las otras perspectivas cognitivas y culturales para colonizarlas luego con una forma exclusiva de saber en torno a la cultura europea (Dussel, 1994; Escobar, 2005; Quijano, 2007). Sin embargo, pese a que la modernidad necesitó de la definición de discursos que aún hacen funcionarse por verdaderos y por los únicos que 
deberían ser aceptados (Foucault, 1979), existen racionalidades que pervivieron y que hoy, durante la crisis de la civilización, nos ayudan a entender que los sistemas de pensamiento occidentales han encontrado desde hace tiempo sus límites. No creemos tampoco que existan mejores formas de entender el mundo que otras. Pero en un momento en el que la disyuntiva de la existencia nos ha planteado la pregunta por la posibilidad de seguir siendo sobre la Tierra, es necesario inspirarnos en ontologías relacionales y superar aquellas fragmentarias sobre las que se ha edificado el sistema-mundo capitalista.

Precisamente, sobre el tema de la supervivencia de la especie humana se están elaborando discursos alternativos inspirados en racionalidades vivas que, a nuestro juicio, se dirigen al meollo del asunto. No son esencialismos o romanticismos sobre ciertas culturas, ni intentos de uniformización y universalización de cosmovisiones que, por definición, son heterogéneas y plurales. Más bien son puntos de encuentro donde se reúnen diversas maneras de pensamiento para responder multiculturalmente al desafío de nuestra no asegurada vida en el planeta. En particular, queremos concentrarnos en la propuesta del Buen Vivir, una utopía que está configurándose en Latinoamérica, y que nace inspirada en la filosofía de los pueblos indígenas, campesinos y afro descendientes del subcontinente y, a contrapelo, intenta hilvanar una propuesta alternativa a la esencia de la modernidad misma. El movimiento, entre muchas otras cosas, busca un acuerdo internacional por medio del cual se le declaren derechos a la Madre Tierra.

La finalidad de este artículo es ofrecer un marco conceptual que atienda a la pregunta misma del ser a través de respuestas en torno a la relacionalidad. Antes que dar razones de tipo jurídico, lo que buscamos es acercarnos al entendimiento de las ontologías que dan sustento a este proyecto.

\section{LAS ONTOLOGÍAS RELACIONALES}

En la obra citada, El Ser y el Tiempo, Heidegger desarrolla el argumento de la necesidad de que retomemos la pregunta que interroga por el ser, es decir, aquella por la cual nos cuestionamos el qué somos, pero indagándonos ahora por su sentido. Para el filósofo alemán, si bien la pregunta en la metafísica occidental sí se ha planteado, desde Platón se ha olvidado inmediatamente al considerar al ser con referencia a lo cósico (Heidegger, 2000). La consecuencia de tal olvido, es que la esencia del humano ha estado encubierta. De hecho, su arrogante auto determinación como único sujeto en el mundo y la identificación de todo lo demás como objeto, es la causa de 
su principal ocultamiento (Heidegger, 1996, 1994). Según hemos dicho, en la actualidad una prueba del extravío de la respuesta, es la contestación de la pregunta que inquiere por la posibilidad de la continuidad de la vida humana en la Tierra a través del auxilio técnico. En otras palabras, considerar que los paneles solares, la energía mareomotriz, o los alimentos sintéticos constituyen la respuesta. Con Heidegger creemos, en cambio, que el problema tendría que solucionarse atendiendo el sentido del ser, en cuanto tal, sin identificarlo antes con referencia a las cosas.

No obstante, la dificultad descrita no puede adjudicársele a todas las ontologías sobre la faz de la Tierra, sino a una forma de saber muy particular de Occidente, asociada a las necesidades del capital, la cual hace que respondamos la pregunta por nuestro ser a través de la posesión de las cosas. Por fortuna, existen contestaciones alternativas en diversas regiones del mundo que desarrollan el sentido del ser por medio de la relación con el todo. Particularmente prestamos cuidado a las ontologías de algunas comunidades indígenas, campesinas y afro latinoamericanas, de las cuales se está inspirando el discurso político del Buen Vivir para proponer, entre otras, la declaración universal de los derechos de la naturaleza.

Lo primero que debe comprenderse es que el principio de encuentro entre dichas ontologías ${ }^{4}$, es el supuesto según el cual, todo es dependiente, todo está conectado e interrelacionado con lo demás. Nada de lo que existe puede hacerlo de manera autónoma, porque cada entidad es parte integral de la totalidad (Estermann, 1998). Para estas racionalidades, cada uno de los componentes del cosmos cumple una función necesaria de la cual dependen muchas otras para su existencia (Díaz, 2007). Incluso, concebir que algo pueda existir por fuera de la red de vínculos con el todo, es una idea absolutamente absurda, porque es imposible que ese algo sea si no está relacionado. La ontología relacional puede ser descrita como una infinita y compleja trama de asociaciones inseparables e indisolubles, en donde los seres son comunidades de seres, antes que entidades individuales (Santos, 2010). Sin embargo, la comunidad a la que se hace referencia no es la de un círculo humano, sino la de una comunidad que además de personas, incluye a plantas, animales, agua, aire, cielo y montañas (Huanacuni, 2010). Por eso el ser lo definen por su capacidad de hacer "parte de" y "estar con" otros sujetos de ésta gran comunidad.

Haciendo justicia, la filosofía del irrompible lazo que ata a las personas con la naturaleza, los antepasados, los astros, minerales y herramientas, la debemos remontar a las 
cosmovisiones africanas que permitieron asegurar la supervivencia de los primeros humanos sobre la Tierra. Es el pensamiento de pueblos que se sienten parte del universo, porque entienden que la armonía relacional es el principio básico para la convivencia entre seres humanos y naturaleza (Zapata, 1997). Por ejemplo, para las ontologías bantúes del África central, el muntú es la persona, viva o difunta, que está inherentemente relacionada con los demás entes del cosmos, pues el mundo depende del equilibrio entre las fuerzas vitales que lo componen y que están íntimamente interconectadas (Temples, 1959). Dicha filosofía de supervivencia, de acuerdo con Manuel Zapata, fue la sabiduría que permitió a la diáspora africana esclavizada y expoliada resistir a la ignominiosa explotación a la que fueron sometidos en América. Efectivamente, las etnias afro-descendientes latinoamericanas, herederas de la ontología más antigua del planeta y asentadas en la generosa biodiversidad tropical, construyeron su identidad concibiéndose vinculados con los muertos, las divinidades, la familia y la gran comunidad humana y biótica que los cobija.

El principio de la relacionalidad con el todo no es de manera alguna exclusivo de las ontologías indígenas y afro, sino que tiene una profunda afinidad con algunas sabidurías budistas, como lo es el caso de la doctrina madhyamaka -escuela de la vía media- fundada en la India del siglo II por el monje Nāgārjuna. Para esta doctrina la existencia de cualquier cosa o fenómeno depende de otras cosas y fenómenos, que a su vez, también están condicionados. Tal idea se expresa en el concepto sánscrito pratītyasamutpāda, traducido como origen condicionado, relacionalidad o contingencia. Tal perspectiva hace hincapié en la naturaleza interdependiente de todo lo existente, y en el hecho de que no hay nada que tenga una realidad autónoma de lo demás. La filosofía de Nāgārjuna sostiene que no pude hablarse de un elemento aislado que tenga una sustancialidad por sí y a partir de sí mismo, dado que cada cosa descansa y se origina a partir de otra, la cual por su parte, se origina de otra más, en una cadena infinita de relaciones vacías de cualquier origen primario (Arnau, 2005). En términos ontológicos, lo anterior implica la inexistencia de un "yo" individual y demarcado, lo que en otras palabras quiere decir, que no tiene ningún sentido imaginar un "yo" al margen de los vínculos con lo demás, porque ese "yo" está tan hiperrelacionado que su propio ser se desborda.

Análogamente al pensamiento oriental, en las cosmogonías indígenas y afro el ser no puede vivir sino en constante relación con su comunidad, porque lo que cada uno es, se 
determina por sus vínculos con los demás. No obstante, esto no significa la desaparición del individuo, puesto que la comunidad tampoco podría ser si ella no estuviera integrada por cada uno de sus integrantes. Con mayor precisión corresponde a una dialéctica ontológica, en donde el énfasis está dado en las interacciones que constituyen a cada uno de los seres que conforman la totalidad. Para las ontologías relacionales un individuo solo está perdido, es una nada, porque para ser, debe estar relacionado con el conjunto de seres de la gran comunidad de la que hace parte. En definitiva, cada ser podría definirse tan solo como un miembro más, integrado indisolublemente a una compleja red mutualista. Es un actor que cumple una función específica dentro de una concatenada red de relaciones (Estermann, 1998). Así entonces, el acento ontológico para estos pueblos se concentra en el nudo de vínculos que une a los integrantes de la gran comunidad de la Madre Tierra, la cual, por supuesto, está también conformada por los seres humanos.

Aceptar la metáfora de la Tierra como Madre, implica reconocer que las plantas, los ríos, los mares, los animales, las piedras, el suelo y el subsuelo, son nuestros hermanos pues todos, sin excepción, hemos sido paridos por la Tierra. Pero también que los seres humanos seríamos tan solo una criatura más entre las muchas que componen la naturaleza. Antes que seres racionales, somos entes naturales vinculados por nexos vitales con el conjunto de fenómenos cósmicos (Estermann, 1998). La inexistencia oriental del "yo" podría interpretarse bajo la racionalidad indígena, como una individualidad difuminada: un "nosotros", en donde cada quien, para ser, debe integrarse armónicamente a una colectividad (Lenkersdorf, 2005).

Sin embargo al analizar lo anterior es necesario hacer una importante aclaración: la relacionalidad del todo no permite ningún tipo de centrismo, razón por la cual no podría hablarse de enfoque biocéntrico, ecocéntrico, ó cosmocéntrico, sino de una vasta red de relaciones vacías de todo centro. En realidad, si intentáramos poner el dedo en algún eje medular, nos daríamos cuenta de que al hacerlo, estaría en tal sobreabundancia de relaciones que cada cosa podría ser centro y circunferencia en el mismo momento. Llevar tal racionalidad al extremo nos permite dar cuenta de que el fenómeno de la vida no puede pensarse desligado de lo que comúnmente es aceptado como "muerto", pues cada individuo necesita de las relaciones con el agua, el aire ó el suelo que produce el alimento para poder ser. Pero aún más, pensando la vida como un intrincado tejido de relaciones en donde la existencia es el resultado de condiciones hilvanadas en una 
conexión inseparable, tendríamos que preguntarnos ¿qué podría llamarse esencialmente muerto?

La respuesta para los pueblos indígenas es contundente: nada está muerto, todo vive y todo es necesario para el equilibrio y la vida (Huanacuni, 2010). Es la totalidad de la Madre Tierra, la Pachamama, en cuanto universo espacio temporal en donde se interrelaciona la vida en nuestro planeta, un sujeto vivo en toda su gloria y majestad.

El sabio juicio tribal, de que la Tierra es un organismo vivo, no solo atañe a las racionalidades indígenas, sino que es un discernimiento con el que concurren algunos investigadores como es el caso del reconocido científico James Lovelock (2007) con su teoría de Gaia. Según Lovelock, Gaia es un sistema fisiológico que ha sido capaz de mantener la Tierra apta para la vida durante más de tres mil millones de años, dada su facultad de regular su temperatura y su química. Según Lovelock, la biósfera mantiene una homeostasis lograda por la armónica interrelación de los componentes biológicos y físicos que la componen.

Por cualquier vía, ya sea a través de las cosmovisiones indígenas 0 los aportes del conocimiento científico, entender que la Tierra es un organismo vivo nos permite tener una concepción ontológica diferente a la construida durante la modernidad, pues los humanos, como todos los demás sujetos de la Madre Tierra, somos entes en constante relación dentro de un organismo vivo, en donde no existen centros ni periferias. Pero como la pregunta caída en el olvido y la que debe hacerse no es aquella que interroga por el ser, sino por el sentido del ser (Heidegger, 1971), una hermenéutica ecológica del sujeto tiene que efectuarse a través de contestaciones que resuelvan dicho cuestionamiento por la capacidad de integrarse armónicamente a la gran comunidad viva de la Madre Tierra. Si al decir de Heidegger, el ser de los humanos consiste en estar referido a posibilidades, la complicación hoy más grande, es la probabilidad de no continuar siendo posibles. Resolvernos, por tanto, consiste en encontrar nuestra posición específica en la totalidad de relaciones dentro de la Madre Tierra.

Para las racionalidades del Buen Vivir de las comunidades indígenas de los Andes, la posición que hombres y mujeres desempeñamos en el mundo es esclarecedora: el ser humano es ante todo un cuidador, un agri-cultor, en la máxima expresión del término: un cultor responsable de la continuación de la vida (Estermann, 1998). De manera similar, otra respuesta ontológica edificante puede encontrarse en algunas racionalidades de ciertos 
campesinos, para quienes el vínculo con la tierra es el sentido mismo de su propia vida. Es una forma de ser y estar en el mundo, en el que la tierra cultivada les ofrece los alimentos necesarios para vivir y, en correspondencia, ellos actúan de modo recíproco a través de su cuidado. Si la auténtica esencia humana ha estado extraviada, y la soberbia auto-constitución de creernos los únicos sujetos sobre la Tierra fue la principal causa de su ocultamiento, la modesta interpretación de jardineros, cultores, pastores, o cuidadores de un interrelacionado sistema vivo, es quizá la respuesta que más necesitamos para continuar siendo posibles.

Con base en la interpretación descrita creemos que podemos entender, con mayor profundidad, los presupuestos ontológicos del proyecto impulsado por los movimientos del Buen Vivir que explicaremos a continuación.

\section{LA DECLARACIÓN UNIVERSAL DE LOS DERECHOS DE LA MADRE TIERRA}

Para comenzar es preciso mencionar que lo ideal sería que la experiencia humana vivida desde el lugar permitirá la valoración intrínseca de la Tierra. Que existiera un cambio ontológico de un ser humano individualizado y separado de la naturaleza, por uno que, retornando a las raíces de la Tierra, revalorice el fenómeno de la vida y se conciba como parte de la misma. La idea finalmente es que se impulsara la conservación no por altruismo -o por un "deber ser" moral- sino porque el mismo hecho de hacerlo se vea como parte del interés de su propia existencia, según propone la ecología profunda de Arne Naess (2006). Consideramos que apelar a una transformación de tal tipo es ineludible y tiene que partir de un proyecto educativo inscrito a su vez en un proyecto político integral a largo plazo- como tendría que hacerlo desde ya la utopía del Buen Vivir.

Pero en vista de la rapidez con que ha devenido la devastación ambiental no es posible esperar un cambio ontológico de este tipo. Por eso consideramos loable que las racionalidades de ciertas sociedades rurales ayuden a entretejer la construcción de alternativas políticas creativas como reconocerle derechos a la Madre Tierra, hecho que ha ocurrido recientemente en la Constitución ecuatoriana y la legislación boliviana.

Se trata de normativas que aceptan que el ser no es solo el ser de los humanos, sino que la Madre Tierra, en cuanto sujeto vivo, es un ser que tiene derecho a existir. Habrá 
quienes afirmen que otorgarle derechos a la naturaleza es un acto en sí mismo antropocéntrico, porque son los humanos los que se dan el lugar de decidir quién es, o no, sujeto de derechos. Por supuesto que la Madre Tierra no los tiene; ella simplemente es. Ese tema es una invención exclusiva de nuestra especie y carece completamente de sentido para un insecto, un árbol o el agua. Pero no podemos pretender salirnos de nuestros propios zapatos para intentar mirar con objetividad el mundo, porque la realidad emerge dependiente de quien la percibe. Las personas siempre aprehenderemos con las categorías que hemos elaborado culturalmente, como el pensar en términos de derechos. Lo que si podemos hacer desde nuestra posición -de la que es imposible desentendernoses no considerarnos centro de nada, sino entes constitutivamente relacionales $\mathrm{y}$, por tanto, en continua reciprocidad con el medio con el que interactuamos.

En verdad, los derechos de la Madre Tierra pueden ser vistos como un acto de reciprocidad, circunscrito dentro de una normativa relacional, porque en toda relación hay un vínculo bidireccional entre entes ontológicamente diferentes, pero interdependientemente complementarios. En otras palabras no es posible que una de las partes dé y la otra solo reciba -como en el caso de la actividad extractiva- sin que se rompa el equilibrio que debe ser mantenido (Estermann, 1998). En tal sentido, en la misma proporción en que la Tierra nos ofrece todos los elementos físicos, identitarios, afectivos que necesitamos para vivir (Walsh, 2009), ella requiere que los humanos actuemos en correspondencia. De manera que reconocer derechos es una forma de aceptar que, en la simbiosis de la realidad, somos incompletos y requerimos que los demás existan para complementarnos (Medina, 2008) y permitir así nuestra propia existencia.

Los primeros pasos significativos en los que se adoptan estas racionalidades, son los procesos políticos del Buen Vivir que se viven en Latinoamérica. En el caso que nos ocupa, la Carta Magna de Ecuador aprobada en 2008, promulga que la Pachamama: "tiene derecho a que se respete integralmente su existencia y el mantenimiento y regeneración de sus ciclos vitales, estructura, funciones y procesos evolutivos...". El hecho de aceptar que se respete su existencia, ontológicamente significa que se reconozca su ser mismo, ó sea, admitir el derecho de que un bosque, un río, y una montaña, sigan siendo un bosque, un río y una montaña. Además, a contracorriente del proyecto moderno, la Madre Tierra legislativamente no podrá ser considerada más en forma de objeto, sino como un sujeto, más allá de cualquier valoración humana en cuanto a utilidad, interés económico o estético (Gudynas, 2009). 
Por su parte, Bolivia, a través de la Ley de la Madre Tierra, estableció los derechos de la naturaleza, con los cuales se le reconoce el derecho a la vida; a la diversidad; al aire limpio; al agua pura; al equilibrio; a la restauración y a vivir libre de contaminación. Lo más significativo de esta ley es que ubican en el mismo nivel de importancia los derechos de la naturaleza y los humanos. Más allá de los proyectos desarrollistas que quieran implantar los gobiernos, se están creando herramientas jurídicas para que cualquier persona, comunidad u organización pueda exigir el cumplimiento de los derechos de la Madre Tierra.

Es claro que pese a la relevancia de estas dos normativas, es absolutamente insuficiente que dos países aisladamente le reconozcan derechos a la naturaleza. Por eso, luego de finalizar la XV Conferencia de las Naciones Unidas sobre el Cambio Climático en Copenhague 2009, Bolivia convocó la Primera Conferencia Mundial de los Pueblos sobre el Cambio Climático, evento durante el cual se redactó el proyecto de declaración universal de los derechos de la Madre Tierra:

Nosotros, los pueblos de la Tierra...-dice el preámbulo del acuerdo- considerando que todos somos parte de la Madre Tierra, una comunidad indivisible vital de seres interdependientes e interrelacionados con un destino común; reconociendo con gratitud que la Madre Tierra es fuente de vida, alimento, enseñanza, y provee todo lo que necesitamos para vivir bien...convencidos de que en una comunidad de vida interdependiente no es posible reconocer derechos solamente a los seres humanos, sin provocar un desequilibrio en la Madre Tierra; afirmando que para garantizar los derechos humanos es necesario reconocer y defender los derechos de la Madre Tierra y de todos los seres que la componen, y que existen culturas, prácticas y leyes que lo hacen...proclamamos esta Declaración Universal de Derechos de la Madre Tierra, y hacemos un llamado a la Asamblea General de las Naciones Unidas para adoptarla, como propósito común para todos los pueblos y naciones del mundo... (Cmpcc, 2011)

Como puede apreciarse, el texto advierte que así como los seres humanos tienen sus derechos, los demás seres de la Madre Tierra también los tienen, porque al comprendernos como seres intrínsecamente relacionados, no puede haber 
derechos para nadie si no los hay para todos ${ }^{5}$. Por tanto para que puedan garantizarse el derecho a la vida, a la salud, a la libertad, a la dignidad, a los alimentos y el agua para los seres humanos, es indispensable primero proteger los derechos de la Madre Tierra. Considerar que dar prioridad a la Tierra sobre las personas puede dar lugar a un ecofascismo, según se ha sugerido, es no concebir que somos seres dependientes e integrantes de la naturaleza. Por el contrario, un humanismo radical consentiría que únicamente al reconocer los derechos de la Madre Tierra podría ser posible garantizar los derechos humanos.

El proyecto de los pueblos sostiene que en caso de conflictos entre los derechos de cada ser, ellos tendrían que resolverse de una manera que siempre "mantenga la integridad, equilibrio y salud de la Madre Tierra". Es decir, el criterio para decidir sobre los derechos económicos, sociales y culturales de las sociedades humanas debe responder al principio de la armonía y la salud, entendida como un estado de equilibrio biológico entre los miembros de la gran comunidad.

Basándose en las racionalidades indígenas y la teoría de Gaia, en el acuerdo a la Madre Tierra se le considera un sujeto: “...un ser vivo... una comunidad indivisible y auto regulada de seres interrelacionados que sostiene, contiene y reproduce a todos los seres que la componen". El acuerdo continua señalando que "cada ser se define por sus relaciones como parte integrante de la Madre Tierra". La redacción es absolutamente vehemente al aclarar que: "El término "ser" incluye los ecosistemas, comunidades naturales, especies y todas las otras entidades naturales que existen como parte de la Madre Tierra" (Cmpcc, 2011). Sin duda, corresponde a un discernimiento ontológico por el cual no se designa al ser directamente con el "yo" de los humanos, y en cambio se hace una definición ampliada del ser, al considerar a cada uno de los miembros de un organismo vivo.

Teniendo en la cuenta todo lo anterior, la declaración del movimiento de los pueblos por la Madre Tierra subraya que todos los seres tienen derecho a existir y a ser respetados; a la libre auto-regeneración de procesos vitales sin alteración humana; a la identidad en cuanto individuos diferenciados pero interdependientes; al agua; al aire limpio; a la salud integral; a estar libre de contaminación; a no sufrir modificaciones genéticas; a la restauración de las afectaciones producidas por la humanidad; y, a vivir libres de trato cruel por parte de los seres humanos (Cmpcc, 2011). Reconocer estos derechos es asumir nuestra responsabilidad como cultores, cuidadores y pastores, por lo que en el apartado de obligaciones se advierte que 
"todos los seres humanos son responsables de respetar y vivir en armonía con la Madre Tierra" y se conmina a una serie de obligaciones que deben asumir los Estados, organizaciones públicas y privadas al adoptar el acuerdo.

Dijimos que la intención no era permanecer en una meditación filosófica. La idea es que al considerar el ser en cuanto tal, tengamos presupuestos ontológicos que nos sirvan, entre otras cosas, para estimular la creatividad política. Sabemos que la aprobación de la declaración universal de los derechos de la Madre Tierra es ya de por sí un complicado asunto, pues interfiere con los directos intereses del capitalismo. Pero siendo realistas debemos ser aún más ambiciosos y no limitarnos a un acuerdo internacional. Hay que lograr un cambio, una emancipación en contra de nuestros propios pensamientos, para poder sentirnos parte de la naturaleza y asumir las responsabilidades en los actos que dicha transformación ontológica nos obliga.

\section{CONCLUSIÓN}

Comenzamos el artículo mencionando la miseria del pensamiento como causa y consecuencia de la actual crisis civilizatoria. Del hecho de no considerar desde una perspectiva del ser el peligro que la humanidad corre a consecuencia de la "ecocida" civilización construida. Por eso quisimos volver a la pregunta olvidada, aquella que interroga por el sentido del ser en cuanto tal, pero a partir de ontologías relacionales. En divergencia con la concepción lineal y progresiva del tiempo, creemos necesario volver a la conciencia de los primeros habitantes sobre la Tierra quienes permitieron su propia supervivencia al considerarse como entes vinculados en un nudo inquebrantable con los demás seres de la Madre Tierra. Más allá de un misticismo, la apuesta de sentirnos hermanados con los difuntos, como en el muntú africano o las racionalidades indígenas, representa hacer una apuesta por el retorno de lo posible, la vuelta a una concepción ecológica y primigenia de la especie necesaria para sobrevivir, como lo enseñó por generaciones la connotación afro del humano hermanado armónicamente con el resto de los seres de la Tierra y el cosmos (Zapata, 1997).

No quiere decir que pretendamos volver a un estado primitivo. Eso no es viable. Se trata de entender que en la actualidad la hecatombe ambiental es el resultado de la pobreza de pensamiento de la modernidad occidental, y 
que debemos aceptar el reto de transgredir las lógicas predatorias heredadas, y concebirnos de manera semejante a las ontologías relacionales que perviven aún en muchas culturas. Finalmente, lo que está en juego es la posibilidad de que podamos mantener nuestra propia vida en el planeta.

\section{BIBLIOGRAFÍA}

- Adorno, Theodor y Horkeimmer, Max. (1987). Dialéctica del iluminismo. Traducción de Murena H.A. Buenos Aires: Editorial Sudamericana. p. 302

- Arnau, Juan. (2005). La palabra frente al vacio. Filosofía de Nāgārjuna. México D.F.: Fondo de Cultura Económica. Colegio de México. 347 p.

- Cmpcc. (2011). Conferencia Mundial de los Pueblos sobre el Cambio Climático y los Derechos de la Madre Tierra [En línea] Recuperado de http://cmpcc.wordpress.com/2010/04/24/conclusionesfinales-grupo-de-trabajo-3-derechos-de-la-madretierra/\#more-1816 [Consulta: 18 de diciembre de 2012].

- Díaz, Floriberto. (2007). Floriberto Díaz. Escrito. Comunalidad, energía viva del pensamiento Mixe. Robles, S. y Cardoso, R. Comp. México D.F.: UNAM. p.435

- Dussel, Enrique. (1994). 1492: El encubrimiento del otro. Hacia el origen del "mito de la modernidad". La Paz: Plural Editores. p.186

- Escobar, Arturo. (2005). Más allá del tercer mundo. Globalización y diferencia. Bogotá: Editorial Norma. p.276

- Estermann, Josef. (1998). Filosofía andina. Estudio intercultural de la sabiduría autóctona andina. Quito: Ediciones Abda-Yala. p. 359

- Foucault, Michel. (1979). Microfísica del poder. Traducción de Varela, J. y Álvarez, F. Madrid: Las Ediciones de la Piqueta. p.189

- Gudynas, Eduardo. (2009). La ecología política del giro biocéntrico en la nueva Constitución de Ecuador. Revista de Estudios Sociales. 32: 34-47.

- Heidegger, Martin. (1971). El ser y el tiempo. Traducción de Gaos, J. México D.F.: Fondo de Cultura Económica. p.478

- Heidegger, Martin. (1994). La pregunta por la técnica. Barcelona: Ediciones del Serbal. Traducción de Barjaú, E.

- Heidegger, Martin. (1996). La época de la imagen del mundo. En: Caminos del Bosque. Traducción de Cortés, $\mathrm{H}$ y Leyte, A. Madrid: Alianza.

- Heidegger, Martin. (2000). Carta sobre el humanismo. Traducción de Cortés, H. y Leyte, A Madrid: Alianza Editorial.

- Huanacuni, Fernando. (2010). Buen Vivir/Vivir Bien. Filosofía, políticas, estrategias y experiencias regionales 
andinas. Lima: Coordinadora Andina de Organizaciones Indígenas. p. 74

- Lenkersdorf, Carlos. (2005). Filosofar en clave tojolobal. México D.F.: Miguel Ángel Porrúa. p. 277

- Lovelock, James. (2007). La venganza de la tierra. Traducción de García, M. México, D.F.: Editorial Planeta. p. 249

- Medina, Javier. (2008). Suma Qamaña. La comprensión indígena de la Buena Vida. La Paz: GTZ. p. 238.

- Naess, Arne. (2006). El movimiento de ecología profunda: algunos aspectos filosóficos. En: Los caminos de la ética ambiental. Kwiatkowska, T. e Issa, J. Comp. México D.F.: Plaza y Valdez Editores. p. 321

- Quijano, Anibal. (2000). Colonialidad del poder, eurocentrismo y América Latina. En: La colonialidad del saber: eurocentrismo y ciencias sociales. Perspectivas latinoamericanas. Lander, E. (Comp.) Buenos Aires: CLACSO. p.246

- Santos, Boaventura de Sousa. (2010). Refundación del Estado en América Latina: perspectivas desde una epistemología del sur. México D.F.: Siglo XXI editores, Siglo del Hombre Editores, Universidad de los Andes. p.186

- Temples, Placide. (1959). Bantu Philosophy. Traducción al inglés por Rubbens, A. Paris: Présence Africane. p.127

- Walsh, Catherine. (2009). Indigenous and afro struggles and the State: Interculturality, decoloniality, and collective well being in the Andes. En: Lozano Long Conference, "Contested Modernities". Universidad de Texas,Austin.

- Zapata, Manuel. (1997). La rebelión de los genes. El mestizaje americano en la sociedad futura. Bogotá: Altamir Ediciones. p. 368

1. Agradezco las sugerencias y comentarios críticos de la Dra. Gabriela Kraemer Bayer y del Dr. Guillermo Torres Carral del Departamento de Sociología Rural de la UACh. Los problemas que aún persisten en el texto son de mi total responsabilidad.

2. Doctorante en Ciencias Agrarias. Departamento de Sociología Rural. Universidad Autónoma Chapingo, México. Magister en Desarrollo Rural por la Universidad Nacional de Costa Rica y Médico Veterinario egresado de la Universidad Nacional de Colombia. omarfgiraldo@hotmail.com

3. Al usar el término "existenciaria" parafraseamos a Heidegger, quien hace una diferenciación con la palabra "existencial", para dar cuenta del ser que se pregunta por el sentido de su existencia.

4. Reiteramos una vez más que si bien la utopía se apoya en cosmovisiones y filosofías de culturas diversas, no pretende equipararlas, y borrar de tajo sus distintivos y características propias. Si bien la trampa es caer en una excesiva 
generalización, la idea es hacer un diálogo intercultural del cual podamos inspirarnos para la creatividad política.

5. Es importante aclarar, que cuando se hace referencia a los derechos para todos se está hablando de la totalidad de la Madre Tierra y no a sujetos individuales como podría malinterpretarse. La idea es que las acciones humanas no violen las congruencias del mundo natural. 